\title{
Sturge Weber Syndrome - A case Report
}

\author{
A K Mehta,President SMB C/O Military Hospital Bhopal - 462031
}

\section{INTRODUCTION}

Sturge Weber Syndrome is a rare clinical entity $S$ diagnosed clinically by combination of facial portwine stain and epilepsy. The oral and nasal mucosae may be involved with vascular naevi of portwine type. Here we present and discuss one such rare case.

\section{Case Report}

A 25 years old male patient presented to the ENT OPD with reddish discolouration of $(R)$ side of the throat which he noticed when he looked into a mirror. He had no complaints of fever, cough, sore throat or hoarseness of voice. He had been to many ENT surgeon for this apparently inflammed mucesa and had been treated with antibiotics, antihistamines etc with no relief. Examination showed reddish discolouration or oropharyngeal mucosa on $(R)$ side involving the anterior and posterior tonsillar pillars (R) tonsil and (R) half of soft palate upto the uvula. There was no mucosal oedema, displacement of tonsils or trismus, indirect laryngoscopy was normal. Nasal and aural examination was normal. On general examination he was noticed to have unilateral portwine staining of $(R)$ side of face extending from pinna upto the chin (Fig1). Based on this finding of oropharyngeal and cutaneous staining diagnosis of sturge Weber Syndrome was considered. On direct questioning the patient gave history of occasional epileptic fits. He was investigated further. Ocular examination did not reveal any abnormality. Xray skull and CT scan of skull showed cortical calcifications. EEG showed reduced cortical activity. This confirmed the diagnosis of Sturge Weber Syndrome. Patient was counselled regarding his pharyngeal problem, placed on anticovulsants for epilepsy and advised regu- lar ocular examination to detect early changes of glaucoma.

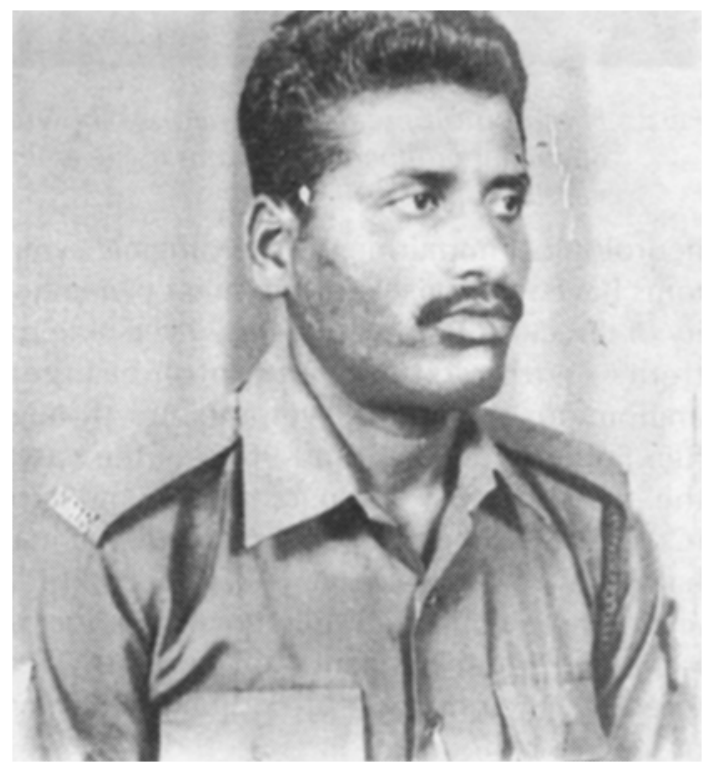

Fig 1: Showing Portwine Staining of Face.

\section{Discussion}

Sturge Weber Syndrome is a developmental malformation of the vasculature of the facial skin and leptomeninges. Convincing evidence of genetic aetiological factor is lacking but there is report of its occurence in monovular twins (Jacobs $\mathrm{AH}$ 1987).

The usual cutaneous finding is unilateral portwine staining involving the areas of maxillary and opthalmic divisions of trigeminal nerve. The oral and nasal mucosae may be involved and the lips may be greatly swollen. There is no correlation between extent of port wine stain and degree of leptomeningeal angiomatosis or 


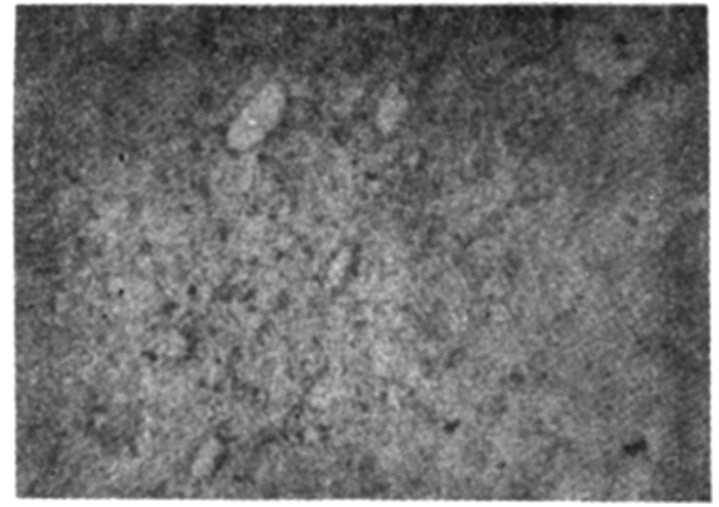

Fig 2: Histipathological examination showing abundant proliperating fibrous tissue amidst vascular spaces

neurological impairment. Neurological symptoms have their onset during infancy in majority of the cases (Lund 1979) howeversome patients with extensive leptomeningeal angiomatosis remain asymptomatic through life. Epilepsy occurs in $80-90 \%$ of the cases, the fits are most often of grand mal type (Gorman 1987). The eye is involved in at least half of all cases (Barkan 1987). A choroidal angioma or congenital glaucoma may occur. Increased iridal pigmentation may be a feature and angiomatosis of conjunctive, sclera or iris maybe found.

In some cases it may be associated with macrocephaly (Crosley 1978) and in other cases by ipsilateral facial hemihypertrophy. In-

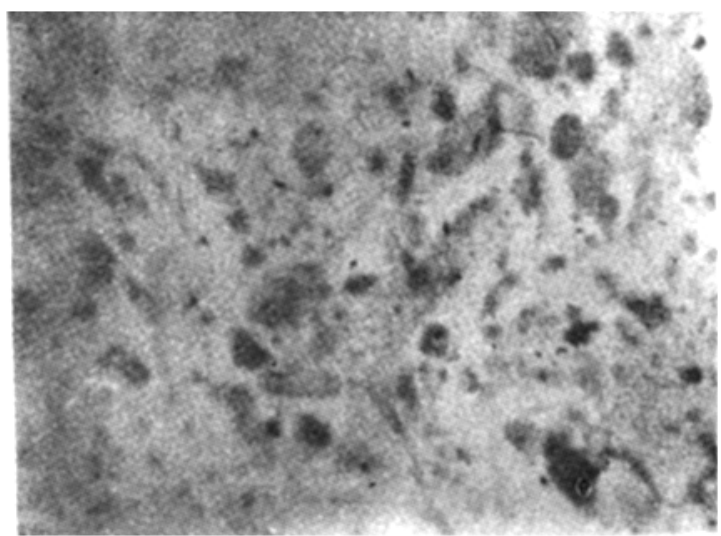

Fig 3: High power examination showing giant cells amongst proliferating fubrous tissue.

vestigations can help to establish leptomeningeal angiomatosis, Cortical calcifications may be seen radiological (Enzmann 1987).

EEG may show suppressed activity with focal epileptiform discharges. Cerebral angiography and $C T$ can are helpful to localize the angiomatosis.

Surgical treatment may be required for oral mucosal overgrowths. Management of fits is by anticonvulsants and neurosurgical intervention where medical treatment does not secure adequate control. The eyes require regular examination by an ophthalmologist to detect earliest changes of glaucoma.

\section{References}

1. Barkan O (1987) : Americal Journal of Ophthalmology, 43, 545.

2. Crosley C J, Binet E E (1978) : Clinical Paediatrics 17, 606.

3. Enzmann D R (1987): Radiology 122, 721

4. Gorman R / (1987): Paediatrics 60, 785.

5. Jacobs $A H$ (1987): Paediatrics 60,788.

6. Lund $M$ (1979): Acta Psychiatrica 24, 569. 